\title{
Needle Steering and Model-Based Trajectory Planning
}

\author{
S.P. DiMaio and S.E. Salcudean \\ Department of Electrical and Computer Engineering \\ University of British Columbia, Vancouver, Canada \\ \{simond, tims\}@ece.ubc.ca
}

\begin{abstract}
Needle insertion for percutaneous therapies is formulated as a trajectory planning and control problem. A new concept of needle steering is developed and a Needle Manipulation Jacobian is defined using numerical needle insertion models that include needle deflection and soft tissue deformation. This concept is used in conjunction with a potentialfield-based path planning technique to demonstrate needle tip placement and obstacle avoidance. Results from open loop insertion experiments are provided.
\end{abstract}

\section{Introduction}

Precise needle placement is important for a number of percutaneous interventions in anaesthesia, biopsy, brachytherapy, etc. Complications associated with poor placement have been studied (see references in [1]2]), and can include tissue damage, misdiagnosis, poor dosimetry and tumor seeding. Straight-line needle trajectories are typically used, likely due to limited needle tracking and the absence of knowledge of steering mechanics required to make trajectory compensation feasible. Mechanical steering mechanisms have been developed for catheters [3]. Needle placement planners for prostate brachytherapy and biopsy have been used to identify optimal seed placement and biopsy sites [4, 5], as well as bone interference [6]; however, misplacement due to tissue deformations and needle deflection have been largely ignored. Kataoka et al. investigated needle deflections during linear insertions into soft muscle tissue ex vivo, and expressed deflection as a function of driving force 7. Tissue deformation and structure were not measured; therefore, their results are difficult to generalise. Alterovitz et al. recently presented a simulation system for analysing the sensitivity of seed placement errors to needle, tissue and trajectory parameters during prostate brachytherapy procedures, using a simple linear needle insertion model 8]. Needle deflection was not considered. This paper outlines the problem of motion planning for the insertion of flexible needles into soft tissues, and presents a new motion planning approach. Section 2 introduces model-based needle motion planning and steering, while Section 3 presents a new potential-fieldbased planning technique. Results of open loop insertion experiments, that are based on planned trajectories, are provided in Section 4. Sections 5 and 6 discuss limitations and plans for future work. 


\section{Motion Planning and Needle Steering}

For the sake of exposition, we define the concepts in 2-D space, for which tissue and needle models have already been derived [1,2]. Consider linear elastostatic model approximations for both tissue and needle. A suitable discretisation, based on Finite Element Analysis (FEA), yields sets of tissue nodes $n_{t}$ and a needle nodes $n_{n}$, locations that determine the tissue-needle configuration $q$ [1. Through the model, the displacements of the tissue $\underline{u}_{t}$ and needle $\underline{u}_{n}$ are related to applied tissue forces $\underline{f}_{t}$ and needle forces $\underline{f}_{n}$, as follows:

$$
\begin{aligned}
\underline{f}_{t} & =F_{t}\left(\underline{u}_{t}\right) & \underline{u}_{t} & =A \underline{u}_{n} \\
\underline{f}_{n} & =F_{n}\left(\underline{u}_{n}\right) & \underline{f}_{n} & =B \underline{f}_{t}
\end{aligned}
$$

We use Cauchy strain for the tissue model, resulting in a linear function $F_{t}\left(\underline{u}_{t}\right)$; and Green-Lagrange strain for the needle model, resulting in a non-linear function $F_{n}\left(\underline{u}_{n}\right) . A$ and $B$ define the coupling boundary conditions between needle and tissue models, as described in 2. The needle is manipulated from its base. Given a tissue-needle configuration $q$ and needle base velocity $\left\{\dot{x}_{b}, \dot{y}_{b}, \dot{\theta}_{b}\right\}$, a new configuration $(q+\delta q)$ after a time interval $\delta t$ can be computed by (1). The motion planning problem is to find a sequence of control parameters (needle base velocities) that guides the needle tip node $\left(n_{n}\right)_{1}$ to a target tissue node $T=\left(n_{t}\right)_{i}$, as shown in Figure प(b). There may be constraints due to tissue force limits, and displacement constraints to avoid obstacles. We say that a needle is "steerable" if base motion can be used to control the position and orientation of the needle tip with respect to the target. This is illustrated in Figure 1(a). A Needle Manipulation Jacobian $J$ embodies this, and is defined by the relationship between the needle tip and base velocities, $\left(\dot{x}_{t}, \dot{y}_{t}, \dot{\theta}_{t}\right)^{T}$ and $\left(\dot{x}_{b}, \dot{y}_{b}, \dot{\theta}_{b}\right)^{T}$ respectively:

$$
\left.J=\left[\begin{array}{lll}
\frac{\partial x_{t}}{\partial x_{b}} & \frac{\partial x_{t}}{\partial y_{b}} & \frac{\partial x_{t}}{\partial \theta_{b}}
\end{array}\right]\left[\begin{array}{lll}
\frac{\partial y_{t}}{\partial x_{b}} & \frac{\partial y_{t}}{\partial y_{b}} & \frac{\partial y_{t}}{\partial \theta_{b}}
\end{array}\right]\left[\begin{array}{lll}
\frac{\partial \theta_{t}}{\partial x_{b}} & \frac{\partial \theta_{t}}{\partial y_{b}} & \frac{\partial \theta_{t}}{\partial \theta_{b}}
\end{array}\right]\right]^{T}
$$

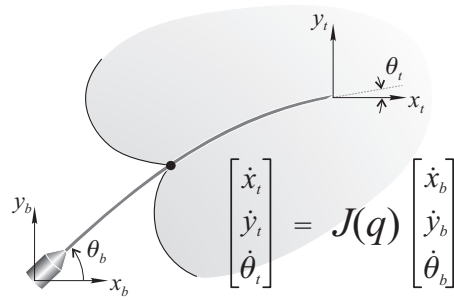

(a)

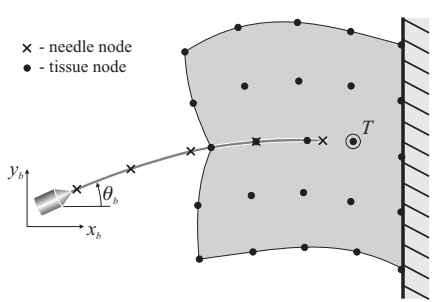

(b)

Fig. 1. (a) Steering and the Needle Manipulation Jacobian. (b) A flexible needle is manipulated from its base, while discrete tissue and needle models characterise motion and force at node points. 
In terms of infinitesimal changes in position, we have:

$$
\left[\begin{array}{c}
\Delta x_{t} \\
\Delta y_{t} \\
\Delta \theta_{t}
\end{array}\right]=J(q)\left[\begin{array}{c}
\Delta x_{b} \\
\Delta y_{b} \\
\Delta \theta_{b}
\end{array}\right], \quad \text { as }\left\|\left(\Delta x_{b}, \Delta y_{b}, \Delta \theta_{b}\right)\right\| \rightarrow 0
$$

A closed-form expression for $J$ is not available due to the complexity of needletissue interaction (11); however, needle tip displacements, caused by perturbations $\left[\begin{array}{lll}\Delta x_{b} & \Delta y_{b} & \Delta \theta_{b}\end{array}\right]$ applied to the needle base, can be measured to numerically determine $J$. As long as $J$ is well conditioned, the tip velocity can be controlled by the base velocity. This means that $J^{-1}$ exists and Equation (2) can be inverted in order to compute the needle base motion required to steer the tip.

\section{Needle Motion Planning Using Potential Fields}

The needle insertion problem exhibits a large number of degrees-of-freedom, corresponding to an extremely high-dimensional configuration space; therefore, global search methods are not feasible. A local search method, based on potential fields, is demonstrated in this section. Consider the simple tissue domain illustrated in Figure 2(a), and the problem of guiding the needle tip from an initial position to the target, while avoiding the obstacle. The needle tip configuration space $\mathcal{C}$ is parameterised by the position and orientation of the needle tip.

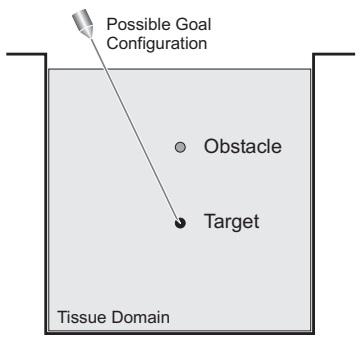

(a)

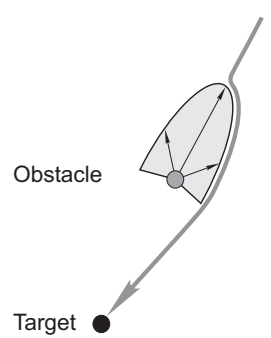

(b)

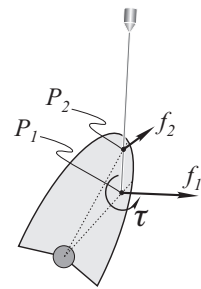

(c)

Fig. 2. (a) A simple 2D tissue domain that contains a target and an obstacle. (b) A hemi-elliptical repulsive field. (c) Repulsive forces at two points near the needle tip generate a "torque".

\subsection{The Attraction Potential}

For an approach based on potential functions, an attractive parabolic potential well $\check{U}$ is placed at the target $[9]$ :

$$
\check{U}(q)=\frac{1}{2} k\left\|q-q_{\text {goal }}\right\|^{2} \quad \Rightarrow \quad \check{F}(q)=-\nabla \check{U}(q)=-k\left(q-q_{\text {goal }}\right),
$$

where $k$ is a positive scaling factor and $q \in \mathcal{C}$. The function $\check{U}$ has a minimum at $q_{\text {goal }} \in \mathcal{C}$ and is positive elsewhere, monotonically increasing in all directions away from $q_{g o a l}$. The gradient of this potential function is an attraction force $\check{F}$. 


\subsection{The Repulsion Potential}

A repulsive potential $\hat{U}$, placed around the obstacle region, prevents the needle tip from intercepting an obstacle [9]. This barrier must be carefully selected so that it does not affect needle motion when it is sufficiently far from the obstacle. A simple repulsive potential function may be written as:

$$
\hat{U}(q)= \begin{cases}\frac{1}{2} \eta\left(\frac{1}{\rho}-\frac{1}{\rho_{0}}\right)^{2} & \text { if } \rho \leqslant \rho_{0} \\ 0 & \text { if } \rho>\rho_{0}\end{cases}
$$

where $\eta$ is a positive constant, $\rho$ is the Euclidean distance $\left\|q-q_{o b s}\right\|$, $q_{o b s}$ is the position of the obstacle and $\rho_{0}$ defines a maximum region of repulsion. The scaling factors $k$ in (3) and $\eta$ in (4) are chosen such that the attraction and repulsion potentials are of the same order of magnitude. If the obstacle is not a circular $\backslash$ spherical point object, then $\left\|q-q_{\text {obs }}\right\|$ becomes $\min _{q_{\text {obs }} \in \mathcal{C B}} \| q-$ $q_{o b s} \|$. For a point obstacle, the resulting repulsion force is isotropic within a circular region of influence. Such a field is not feasible for needle motion planning because of the large lateral deviation that may be required at the needle tip. Needle motion becomes increasingly constrained as the needle penetrates further into the tissue (i.e., the condition number of $J$ increases with needle depth). Therefore, needle trajectory compensation must be made early, and is facilitated by selecting an elliptical or hemi-elliptical repulsive potential field shape, as shown in Figure 2(b). The anisotropic, elliptical repulsive force field is described as:

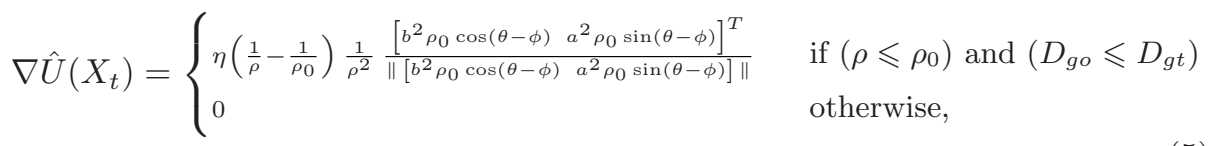

where $a$ and $b$ are the radii at the major and minor axes of the elliptical region, $\phi$ is the angle of the major axis, chosen to be $\angle\left(X_{o b s}-X_{\text {goal }}\right), \rho$ is the Euclidean distance $\left\|X_{t}-X_{o b s}\right\|$, and the region of repulsion $\rho_{0}$ is $\frac{a b}{\sqrt{b^{2} \cos ^{2}(\theta-\phi)+a^{2} \sin ^{2}(\theta-\phi)}}$. $D_{g o}$ is $\left\|X_{\text {goal }}-X_{o b s}\right\|$ and $D_{g t}$ is $\left\|X_{\text {goal }}-X_{\text {tip }}\right\| . X_{o b s}, X_{\text {goal }}$ and $X_{t}$ are the Cartesian coordinates of the obstacle, target and needle tip, respectively. In practice, it is necessary to match the field gradient along the major and minor ellipse axes by scaling both $\rho$ and $\rho_{0}$ by $\frac{a}{\rho_{0}}$ in (5). This defines the translational case. The effect of a repulsive potential field on needle tip heading is included by considering the repulsive force at two points $P_{1}$ and $P_{2}$ on the needle shaft, both close to the tip. This is shown in Figure 2(c). The force "exerted" by the field at each end of the line segment that connects $P_{1}$ and $P_{2}$ results in an effective moment that is used to guide angular motion at the needle tip.

$$
\tau(q)=\left(\frac{X_{1}-X_{2}}{2}\right) \times\left(-\nabla \hat{U}_{1}(q)+\nabla \hat{U}_{2}(q)\right),
$$

where $X_{1}$ and $X_{2}$ are the positions of $P_{1}$ and $P_{2}$, while $\hat{U}_{1}$ and $\hat{U}_{2}$ are the potentials computed at $P_{1}$ and $P_{2}$ respectively. 


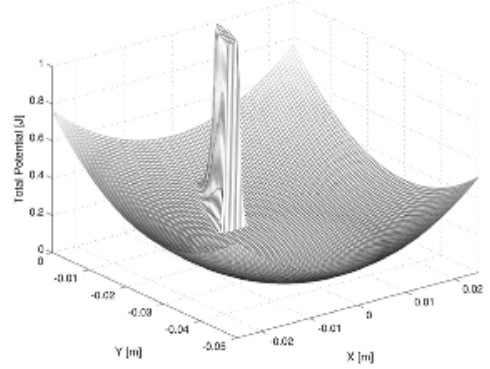

(a)

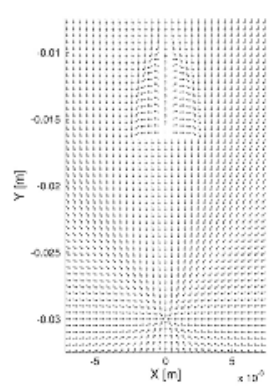

(b)

Fig. 3. (a) Total summed potential over the tissue domain, and (b) the local direction of the field gradient.

\subsection{Minimising Total Potential}

The sum of the attraction and repulsion potentials results in a field that attracts the needle tip toward a goal configuration (tip coinciding with target), while repelling it from obstacles. This is shown in Figure 3. Similarly, multiple obstacles can be accommodated by adding each of their repulsive fields together.

In classical problems that consider path planning for rigid or articulated bodies with mass, the potential field gradient force is often applied and acceleration derived to obtain motion. The notion of mass at a needle tip does not exist; therefore, the force is used to indicate the direction of motion only. Motion of the needle base $\Delta Q_{b}$ can then be expressed as:

$$
\Delta Q_{b}=-J^{-1}\left(W \frac{\nabla \check{U}(q)+\nabla \hat{U}(q)}{\|\nabla \check{U}(q)+\nabla \hat{U}(q)\|}\right)
$$

where $\nabla \check{U}(q)$ and $\nabla \hat{U}(q)$ are attraction and repulsion field gradients respectively, and $W$ is a diagonal weighting matrix.

\subsection{The Planning Algorithm}

The model simulation and trajectory plan updates are computed according to the following algorithm:

1. Select the initial needle configuration.

2. Locate the target and obstacles in the tissue model.

3. Compute the gradient of the potential field at the needle tip, $P_{1}$.

4. Compute the tip heading gradient according to Equation (6) .

5. Set desired tip displacement in the direction of the field gradient.

6. If $J^{-1}$ exists (i.e., $\operatorname{cond}(J) \leqslant \varepsilon$ ), compute the required base displacement by (7), else generate an error condition.

7. Advance the needle base by the displacement (77) and solve (1D to find the new needle $\backslash$ tissue configuration.

8. Go to 2 and iterate until the needle tip and target are coincident. 

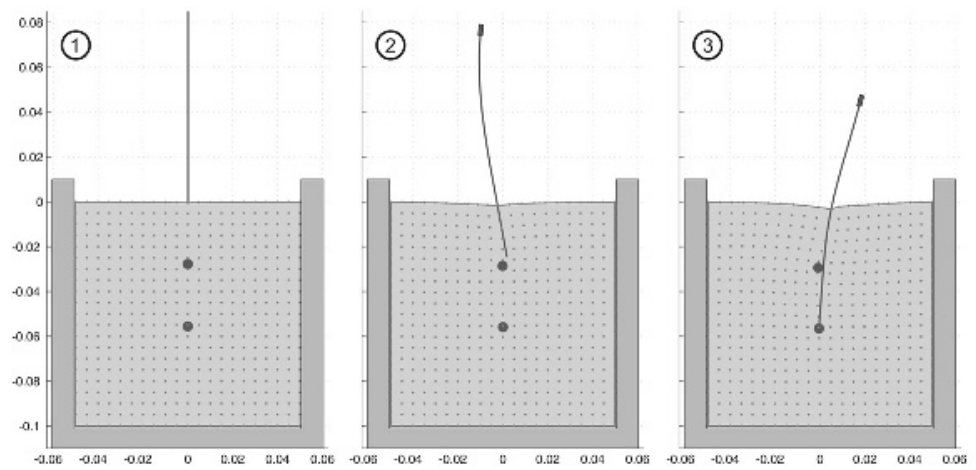

Fig. 4. A simulated needle insertion along a path automatically prescribed by the potential field gradient.

\section{Experimental Results}

Figure 4illustrates a planned trajectory for a $10 \mathrm{~cm}, 22 \mathrm{G}$ Franseen biopsy needle in a soft tissue phantom $(E=20 \mathrm{KPa})$ containing one obstacle and one target as shown in frame 1. The simulated needle trajectory was applied in experiments using the physical measurement system presented in 1, 2. A Franseen tip is used to minimise deflection due to the bevel. Camera images corresponding to frames 2 and 3 in Figure 4 are shown in Figure 5. The measured needle trajectory closely resembles the simulated trajectory; however, this is not immediately apparent from the images, due to out-of-plane deformations. The grid of black markers (visible in the camera images) is applied to the top surface of a $12 \mathrm{~mm}$ thick PVC phantom, whereas the needle moves in a plane that is $6 \mathrm{~mm}$ below this surface. Out-of-plane deformations cause the needle and marker planes to diverge slightly (clearly evident at the insertion point indicated). Based on the magnitude of the divergence at the insertion point, we estimate approximate target and obstacle locations in the needle plane. These are superimposed on the image frames. A second motion planning problem, involving three obstacles, is illustrated in Figure 6. In practice, the planning algorithm is sensitive to the selection of the free parameters $a, b, \rho_{0}$ and $W$. The shapes of the elliptical repulsive fields

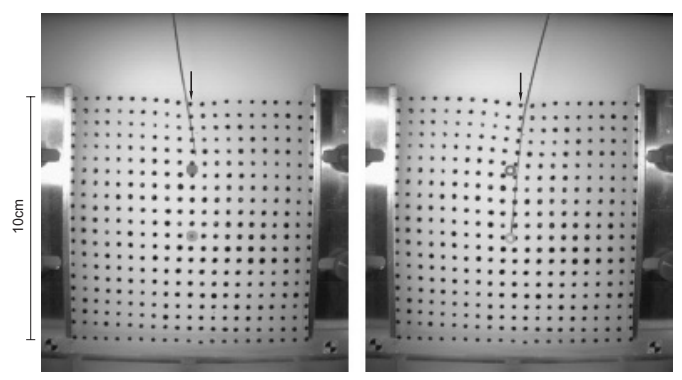

Fig. 5. Robot controlled trajectory in a transparent PVC phantom. The arrows indicate the initial insertion point. Note divergence due to out-of-plane deformations. 

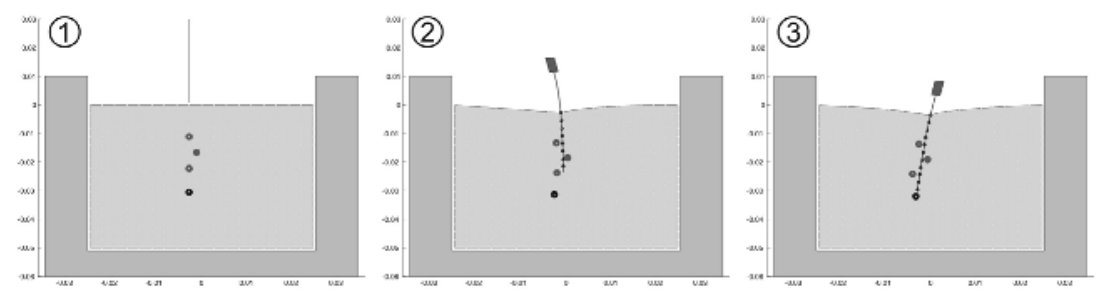

Fig. 6. Simulated needle trajectory plan in an environment containing three obstacles.

are governed by $a$ and $b$, and are chosen so that trajectory adjustments are made early (before reaching the obstacle), and with care not to introduce local minima. The maximum region of repulsion $\rho_{0}$ is selected based on the size of each obstacle. The diagonal weighting matrix $W$ scales the desired tip motion in the direction of the potential field gradient. It accounts for measurement units and the relative importance of motion in each degree of freedom at the needle tip.

\section{Discussion}

A feasible path from the current needle configuration to a goal configuration in each static potential field is not guaranteed to exist, e.g., if the free-space in $\mathcal{C}$ is disjoint. Both the tissue and the needle deform depending upon the path, thus the evolution of the potential function from one time step to the next is very important. Even if a feasible path is shown to exist at each time step, convergence is not guaranteed, e.g., a target that is embedded in soft tissue might be pushed away by the needle as it approaches, and continue to move away as the needle tip is steered toward it. In this case, the manipulation Jacobian $J$ is close to singular, a condition that is easily detected $(\operatorname{cond}(J)>\varepsilon)$. Such a state may be used to indicate that the initial configuration was inappropriate, or that the potential field parameters need adjustment. In some cases, a trajectory may fail to reach the target despite the manipulation Jacobian remaining nonsingular, due to limited achievable needle curvature. Local minima can occur in the potential function, and may be avoided by selecting an alternative set of potential functions. Randomised planning, or hybrid strategies that include some form of configuration space search 9] may also be required in some cases. In addition, elliptical repulsive fields may not be suitable for all obstacle geometries, and may need to be modified for some tissue structures. In general, the existence of solutions to such motion planning problems is difficult to prove. Indeed, such proofs do not even exist for problems with far fewer degrees of freedom.

\section{Conclusion}

The concepts of needle steering and manipulation have been proposed, and a Needle Manipulation Jacobian has been introduced for this purpose. In clinical 
applications, needle steering may be able to avoid obstacles, and reach regions that are currently inaccessible using straight-line trajectories. Geometric constraints and sensitivity analysis for needle insertions have been considered in prior work; however, this paper presents the first needle trajectory motion planning technique that incorporates soft tissue motion, needle flexibility and a physically-based contact model. The high-degree-of-freedom motion planning problem is solved using a task-space potential field approach. The algorithm has been demonstrated in simulation, and validated for simple trajectories in tissue phantoms. Several advancements are required in order to apply these fundamental concepts to clinical applications. Imaging systems that can accurately measure the complex deformation of tissue volumes are required for closed loop control and navigation. Methods for determining tissue parameters in vivo, such as ultrasound and MRI elastography, are still in development. Trajectory analysis, navigation and automatic control are interesting extensions to this work.

\section{References}

1. DiMaio, S.P., Salcudean, S.E.: Needle Insertion Modelling for the Interactive Simulation of Percutaneous Procedures. In: Medical Image Computing and Computer Aided Intervention. Volume 2489. (2002) 253-260

2. DiMaio, S.P., Salcudean, S.E.: Needle Insertion Modelling and Simulation. In: IEEE Transactions on Robotics and Automation: Special Issue on Medical Robotics. (2003)

3. Mineta, T., Mitsui, T., Watanabe, Y., Kobayashi, S., Haga, Y., Esashi, M.: Batch fabricated flat meandering shape memory alloy actuator for active catheter. In: Sensors and Actuators A: Physical. Volume 88. (2001) 112-120

4. Zeng, J., Kaplan, C., Bauer, J., Xuan, J., Sesterhenn, I.A., Lynch, J.H., Freedman, M.T., Mun, S.K.: Optimizing prostate needle biopsy through 3-D simulation. In: Proceedings of SPIE Medical Imaging. (1998)

5. Bauer, J.J., Zeng, J., Sesterhenn, I.A., Moul, J.W., Mun, S.K.: Comparison of Prostate Biopsy Protocols using 3-D Computer Simulation. In: Proceedings of the Pacific Medical Technology Symposium. (1998) 109-114

6. Pathak, S.D., Grimm, P.D., Kim, Y.: Pubic Arch Detection and Interference Assessment in Transrectal Ultrasound Guided Prostate Cancer Therapy.

US Patent 6,027,446 (2000)

7. Kataoka, H., Washio, T., Audette, M., Mizuhara, K.: A Model for Relations between Needle Deflection, Force, and Thickness on Needle Penetration. In: Medical Image Computing and Computer Aided Intervention. (2001) 966-974

8. Alterovitz, R., Pouliot, J., Taschereau, R., Hsu, I.J., Goldberg, K.: Needle Insertion and Radioactive Seed Implantation in Human Tissues: Simulation and Sensitivity Analysis. In: IEEE International Conference on Robotics and Automation. (2003)

9. Latombe, J.C.: Robot Motion Planning. Kluwer Academic (1991) 